\title{
Low Cost Prototyping: Part 1, or How to Produce Better Ideas Faster by Getting User Reactions Early and Often
}

\author{
Stephen Brown \\ De Montfort University \\ The Gateway \\ Leicester LE1 9BH \\ +44 (0)116 2577173 \\ sbrown@dmu.ac.uk
}

\author{
Andreas Holzinger \\ Medical University Graz \\ Auenbruggerplatz 2/V \\ A-8010 Graz \\ +433163853883 \\ andreas.holzinger@ \\ meduni-graz.at
}

\begin{abstract}
Although approaches to User Centered Software Development have existed for almost 20 years a rift still exists between theory and practice. In practice, many software projects are designed at the code level. Almost automatically, the end user the human being - vanishes from the viewpoint of the software developers. Good Usability Engineering combines complex back-end functionalities with a well operated, attractive, effective and efficient user interface, with full regard to efficiency. The first half of this two-part tutorial provides practical guidance on how to resolve web design issues quickly using paper prototypes. You will be introduced to the STAR model (Stage, Types, Aims, Resources) for determining an appropriate prototyping/test strategy and then guided through a series of team based activities to produce a paper based micro Website design. In the second part these designs are tested to provide insights into the thinking and behaviour of end users.
\end{abstract}

\section{Categories and Subject Descriptors}

H.2.2 [User Interfaces]: evaluation/methodology, prototyping, screen design, user-centered design.

\section{General Terms}

Measurement, Performance, Design, Reliability, Experimentation, Human Factors.

\section{Keywords}

Rapid prototyping, design, usability, accessibility, conflict resolution, cost reduction, user-centred design.

\section{INTRODUCTION}

This hands-on tutorial provides practical guidance on how to resolve web design issues quickly using paper prototypes. Delegates will be introduced to the STAR methodology for determining an appropriate prototyping/test strategy and then guided through a series of team based activities to produce and test a paper based micro Website design. Each team will be

(c) The Author 2008.

Published by the British Computer Society given a design scenario and a set of related content resources (images and text). Their tasks will be to:

1. Define some typical site users, by creating user profiles with the aid of a prompt sheet.

2. Determine what the site is for and therefore its intended outcomes when users interact with it.

3. Develop user activities designed to achieve these outcomes and describe the site functionality required.

4. Convert activity descriptions into a paper-based micro Web site design (site map and page layouts) using the content resources supplied, plus scissors, glue and pens.

5. Develop a set of questions and activities for users to carry out to test the performance of their site design.

6. Test their sites on the opposing teams, record the results and report back their findings.

There will be two different design scenarios and teams will be paired so that each team tests an unfamiliar scenario. Team size will range from 3 to 5 delegates, depending on numbers.

\section{KEY QUESTIONS}

The tutorial will address the following key questions:

\subsection{Why build a prototype?}

How good is your design? How can you improve it? How can you avoid disagreements in the design team and fruitless waste of time and effort on designs that are not going to work?

\subsection{What kind of prototype is best?}

What types of prototype are there and what kinds of issues about the design can they uncover? How does prototyping fit into an overall project methodology?

\subsection{How long should a prototype take?}

Which areas of the design should the prototype focus on? Does it have to look professional? Do you need to be an artist to produce good prototypes?

\subsection{How do you build a paper prototype?}

What are we actually testing? What should you model in your paper prototype? How does paper prototyping reduce risk for the design and project as a whole?

3. LEARNING OUTCOMES

By the end of the tutorial delegates will be able to 
- Explain why prototyping early and often is good design practice.

- Describe the advantages and limitations of paper prototypes and their role in the design cycle.

- Create their own paper prototypes, rapidly.

- Test their design ideas using the prototypes they have created.

\section{THEORETICAL BACKGROUND} perform actions that result in achievement of their goals [1]. Goals can be defined in terms of intended outcomes which act prototypes [2]. Prototypes vary according to the breadth or depth of features implemented. Usually we choose between the following types [3]: Vertical Prototype: in-depth functionality for a few selected features; Horizontal Prototype: full interface features, but no underlying functionality; Scenario Prototype: only functionality for specific scenarios or paths through the interface. Paper-based prototyping bypasses the time and effort required to create a working, coded user interface. Instead, it relies on very simple tools like paper, scissors, and stickers [4], [5]. Paper mock-ups only need to capture the site's functionality and convey the right information [6], [7].

\section{STAR}

The model presented in the tutorial considers the design Stage (initial concept, development shell, detailed content); Types of prototypes (paper, wireframes, "look and feel"); the Aims of the evaluation (what you want to test); and the Resources available for testing (time, money, skills).
Successful interfaces minimize cognitive load and help users to as benchmarks for developing and testing functionality through

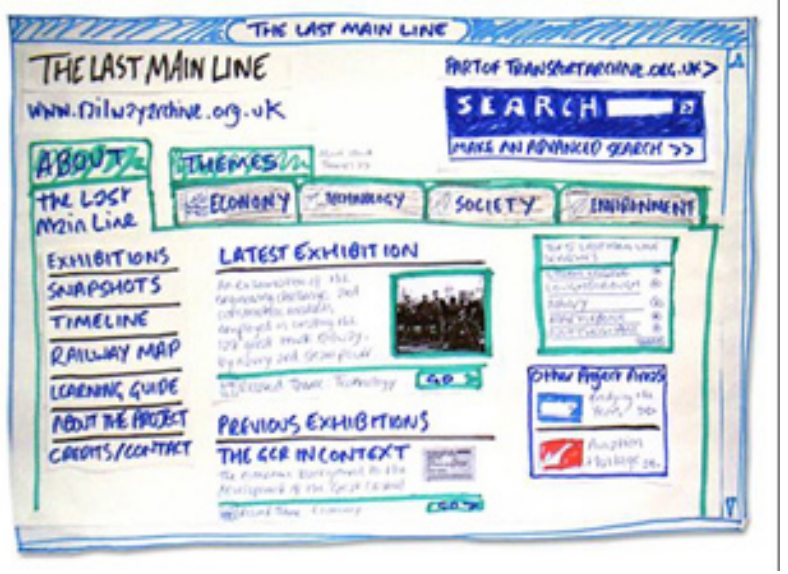

Figure 2. An example paper prototype home page.

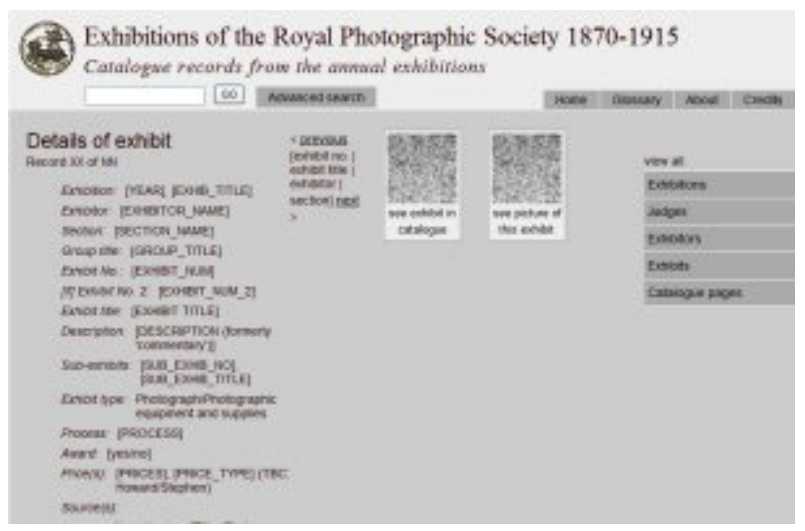

Figure 3. A wireframe mockup showing placeholders.

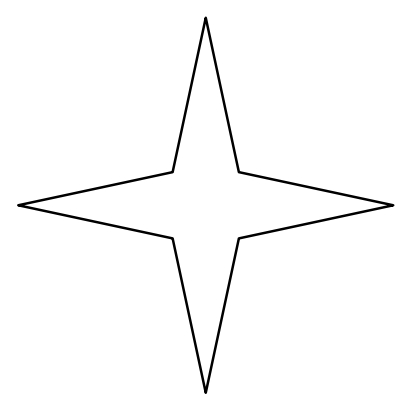

Aims

Resources

Figure 1. The STAR model.

\section{REFERENCES}

[1] Krug, S., Don't Make Me Think! New Riders Publishing, Indianapolis, 2000.

[2] Brown, S. Let's Be Specific: Predicting Return on Investment in On-line Learning Activity Design. In J. Trant and D. Bearman (eds.) Museums and the Web 2007: Proceedings. Toronto: Archives \& Museum Informatics. http://www.archimuse.com/mw2007/papers/brown/brown. $\underline{\mathrm{html}}$

[3] Nielsen, J., Usability Engineering, Morgan Kaufmann, San Francisco, 1993.

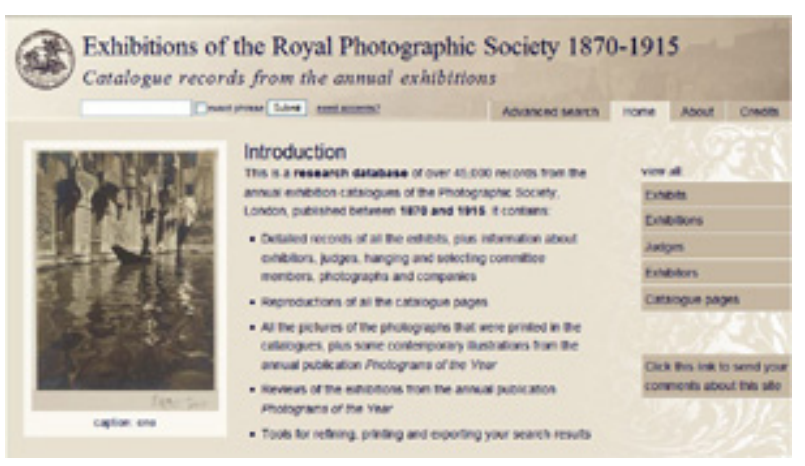

Figure 4. A final visual treatment.

[4] Rettig, M., Prototyping for tiny fingers, Communications of the ACM, 37, 4, (1994), 21-27.

[5] Holzinger, A., Application of Rapid Protoyping to the User Interface Development for a Virtual Medical Campus, IEEE Software, 21 1, (2004), 71-54.

[6] Hix, D. and Hartson, H. R., Developing user interfaces: ensuring usability through product \& process, Wiley, New York, 1993.

[7] Holzinger, A., Usability Engineering for Software Developers, Comm of the ACM, 48, 1, 71-74. 